\title{
Isoliquiritigenin inhibits the proliferation, apoptosis and migration of osteosarcoma cells
}

\author{
CHENGJUN LI* ${ }^{*}$ XING ZHOU* ${ }^{*}$ CHANG SUN, XIAOZHOU LIU, XIN SHI and SUJIA WU \\ Department of Orthopedics, Jinling Hospital, Nanjing University School of Medicine, \\ Nanjing, Jiangsu 210002, P.R. China
}

Received March 28, 2018; Accepted January 16, 2019

DOI: $10.3892 /$ or.2019.6998

\begin{abstract}
The overall survival rate of patients with osteosarcoma has remained unchanged for the last several decades. Therefore, novel drugs for osteosarcoma treatment are required. Isoliquiritigenin (ISL), a natural compound, has been demonstrated to inhibit the growth of various tumors. However, it is unclear whether ISL is able to inhibit the growth of osteosarcoma. In the present study, it was identified that ISL was able to inhibit the growth of the osteosarcoma cell line Saos-2 cells in vitro and in xenograft tumors primarily by attenuating tumor cell proliferation and, cell migration and promoting tumor cell apoptosis. Decreased tumor cell proliferation induced by ISL was associated with downregulation of cyclin D1 and upregulation of p53, p21 and p27. Increased tumor cell apoptosis triggered by ISL was associated with downregulation of apoptosis regulator $\mathrm{Bcl}-2$, upregulation of apoptosis regulator Bax and damaged mitochondrial function evidenced by a low level of ATP-synthesis. In addition, ISL was able to inhibit the migratory capacity of Saos-2 cells by modulating the expression of matrix metalloproteinase (MMP)2 and MMP9. Mechanistic analysis revealed that the tumor growth-inhibitory effect of ISL may depend on the action of ISL on the phosphorylation of PI3K and AKT. However, it remains to be investigated whether the inhibitory effect of ISL on the migration of Saos-2 cells was associated with downregulated PI3K/AKT signaling. Overall, the present
\end{abstract}

Correspondence to: Dr Sujia Wu or Dr Xin Shi, Department of Orthopedics, Jinling Hospital, Nanjing University School of Medicine, 305 Zhongshan East Road, Nanjing, Jiangsu 210002, P.R. China

E-mail: sujia_wu@163.com

E-mail: xin_shin@163.com

*Contributed equally

Abbreviations: ISL, isoliquiritigenin; CDKs, cyclin-dependent kinases; CDKIs, cyclin-dependent kinase inhibitors

Key words: osteosarcoma treatment, ISL, signaling pathway, cell proliferation, cell apoptosis, cell migration study provided evidence for the potential use of ISL against osteosarcoma.

\section{Introduction}

Osteosarcoma, one of the most common bone tumors, prevails particularly in children and adults (1). Worldwide, the estimated number of newly diagnosed patients every year is $~ 1-14$ in one million. Surgical resection combined with chemotherapy is the principle strategy for osteosarcoma treatment (2). Chemotherapeutic drugs including cisplatin and methotrexate have been widely used clinically for patients with osteosarcoma (3). However, these drugs disrupt the DNA synthesis of normal cells in addition to tumor cells, thereby causing severe side-effects. In addition, once tumor cells develop chemoresistance, they are harder to control. Therefore, novel drugs that maximize tumor cell killing in osteosarcoma and minimize normal cell damage are urgently required.

Isoliquiritigenin (ISL), a type of flavonoid, is extracted from the roots of licorice and numerous other plants and foods (4). ISL has been used as a food additive and possesses a number of biological activities including antioxidative stress, anti-inflammatory, anti-viral and anti-angiogenic effects $(5,6)$. Recent studies have focused on the antitumor effects of ISL. A wide spectrum of tumors has been demonstrated to be sensitive to ISL, including glioma, ovarian cancer, hepatoma carcinoma and breast cancer (7-10). However, it is unclear whether ISL may be used as a drug treatment for osteosarcoma.

Accumulating evidence suggests that the PI3K/AKT pathway is the underlying signaling pathway that mediates the antitumor effect of ISL. It has been demonstrated that ISL inhibits the proliferation of the prostate cell line DU145 by decreasing the recruitment of PI3K and the phosphorylation of AKT (11). Studies investigating the effect of ISL on breast cancer have revealed that ISL decreases cell proliferation and induces cell apoptosis by disrupting the PI3K/AKT pathway $(12,13)$. It is generally accepted that the activated $\mathrm{PI} 3 \mathrm{~K} / \mathrm{AKT}$ signaling pathway is one of the most important mechanisms that promotes the development of osteosarcoma (14-16). Drugs targeting the PI3K/AKT pathway have been developed for osteosarcoma treatment, including PI3K pan-inhibitors (16). However, due to their weak solubility, instability and high toxicity, these inhibitors have little potential for clinical application (16). A recent study demonstrated 
that ISL significantly suppressed tumor growth in a xenograft mouse model of endometrial cancer without apparent side-effects (17). Based on these findings, it was proposed that ISL inhibited the progression of osteosarcoma by deactivating the PI3K/AKT signaling pathway.

In the present study, firstly, the effect of ISL on osteosarcoma was investigated in vitro by examining tumor cell proliferation, cell apoptosis and cell migration. Secondly, intracellular molecular alterations in PI3K/AKT signaling upon treatment with ISL were assessed. Finally, the effect of ISL on osteosarcoma growth was assessed in a xenograft mouse model. The present study demonstrated the anti-osteosarcoma effect of ISL, suggesting that ISL may serve as a promising agent for osteosarcoma treatment.

\section{Materials and methods}

Cell culture and treatment. The human Saos-2 cell line and a mouse embryonic osteoblastic cell line (MC3T3-E1) were purchased from the American Type Culture Collection (ATCC; Manassas, VA, USA). Cells were maintained in RPMI-1640 containing $2 \mathrm{mM}$ L-glutamine and $25 \mathrm{mM}$ HEPES supplemented with $10 \%$ fetal bovine serum, and $100 \mathrm{U} / \mathrm{ml}$ penicillin/streptomycin at $37^{\circ} \mathrm{C}$ in a humidified $5 \%$ $\mathrm{CO}_{2}$ atmosphere. ISL was purchased from Sigma-Aldrich; Merck KGaA (Darmstadt, Germany) with a purity of $>98 \%$ and was dissolved in dimethyl sulfoxide (DMSO). Cells were incubated with ISL at various concentrations and time-points. Cells treated with DMSO were set as the control group in the present study.

Tumor xenografts in NOD-SCID mice. A total of 10 female 5-week-old NOD-SCID mice were purchased from the Model Animal Research Center of Nanjing University and maintained in a pathogen-free environment with a constant humidity and temperature at $12 \mathrm{~h}$ light/dark cycle with free access to food and water. The weight of the mice ranged from 19 to $23 \mathrm{~g}$. Saos- 2 cells $\left[1 \times 10^{6}\right.$ cells in $0.1 \mathrm{ml}$ phosphate-buffered saline (PBS) for each mouse] were implanted by subcutaneous injection into the flank of the mice. ISL was dissolved in DMSO and further mixed with corn oil. The mice were randomly divided into a corn oil-treated group (control group) and an ISL-treated group (5 in each). Corn oil and ISL were administered by oral gavage at $50 \mathrm{mg} / \mathrm{kg} / \mathrm{day}$ for 56 consecutive days. The tumor volume and body weight were recorded every day until the animals were sacrificed. The mice were anaesthetized by $1 \%$ pentobarbital (i.p.) at a dose of $50 \mathrm{mg} / \mathrm{kg}$ and sacrificed by cervical dislocation. When the mice were sacrificed, the tumors were removed for weighting and were fixed in $4 \%$ paraformaldehyde solution (4 g/100 ml PBS) or stored at $-80^{\circ} \mathrm{C}$. The max tumor size was $920 \mathrm{~mm}^{3}(0.8 \mathrm{~g})$. All animal experiments were approved by the Committee on the Ethics of Animal Experiments of Nanjing University and performed strictly in accordance with the recommendations in the Guide for the Care and Use of Laboratory Animals of the National Institutes of Health (Bethesda, MD, USA).

Cell viability assay. Cell viability was assessed by a Cell Counting Kit-8 (CCK-8; Beyotime Institute of Biotechnology, Haimen, China). Briefly, cells $\left(3 \times 10^{3}\right.$ cells/well $)$ were seeded in 96-well plates and cultured for $24 \mathrm{~h}$. The cells were incubated with various concentrations of ISL for the indicated time-points. For the cell viability assay, cells were incubated with CCK-8 solution (10 $\mu \mathrm{l} /$ well) for $3 \mathrm{~h}$, and the optical density value in each well was determined using a microplate reader at $450 \mathrm{~nm}$.

Flow cytometric analysis of the cell cycle. The cultured cells were digested, and a single cell suspension was prepared. Cells were fixed in cold $70 \%$ ethanol and stored at $4^{\circ} \mathrm{C}$ overnight. The ethanol was removed by centrifugation $(1,000 \mathrm{x} \mathrm{g}$ for $5 \mathrm{~min})$, followed by staining with propidium iodide (PI; Thermo Fisher Scientific, Inc., Waltham, MA, USA) for $30 \mathrm{~min}$ at $4^{\circ} \mathrm{C}$ in the dark. The cell cycle was analyzed by flow cytometry immediately.

Flow cytometric analysis of cell apoptosis. The cultured cells were collected and a single cell suspension was produced by trypsin digestion and gentle pipetting. An Annexin V-FITC Apoptosis Detection Kit (Thermo Fisher Scientific, Inc.) was used. The cells were incubated with Annexin V-FITC and PI for $15 \mathrm{~min}$ at room temperature in the dark. The apoptosis of the samples was analyzed using a FACSCalibur flow cytometer (Becton-Dickinson, Heidelberg, Germany).

Scratch test. Cells were seeded into 6-well plates $\left(5 \times 10^{4}\right.$ cells/well). When the cells had grown to $\sim 80 \%$ confluence, a sterile pipette was used to scratch a line across each well. Following washing with PBS, cell culture was continued. The scratch was photographed at 0 and $48 \mathrm{~h}$ post-scratching. The width of the scratch was analyzed and assessed using Image-Pro Plus 6.0 software (Media Cybernetics, Rockville, MD, USA). The relative cell migration distance was calculated as follows: (The width at $0 \mathrm{~h}$ in the ISL group-the width at $48 \mathrm{~h}$ in the ISL group)/(the width at $0 \mathrm{~h}$ in the control group-the width at $48 \mathrm{~h}$ in the control group). The cell migration distance in the control group was set as 1 .

Transwell assay. The Transwell assay was performed using Falcon cell culture inserts containing $8-\mu \mathrm{m}$ pore size polyethylene terephthalate membranes (BD Biosciences, Franklin Lakes, NJ, USA). The Saos-2 cells were seeded into the upper chamber $\left(5 \times 10^{4}\right.$ cells/well $)$ and maintained at $37^{\circ} \mathrm{C}$ in a humidified $5 \% \mathrm{CO}_{2}$ atmosphere for $48 \mathrm{~h}$. Non-migrating cells remaining on the upper surfaces were removed while the migrating cells were fixed with methanol and visualized by staining with crystal violet solution. The images were captured by a light microscope (Nikon, Tokyo, Japan).

Western blot analysis. Collected cells or tumor samples were homogenized in radioimmunoprecipitation assay buffer containing phenylmethanesulfonyl fluoride (PMSF) and a phosphatase inhibitor. Following centrifugation $(13,800 \mathrm{x} g$ for $15 \mathrm{~min}$ ), the supernatant was collected, and the protein concentration was determined using a bicinchoninic acid assay kit (Beyotime Institute of Biotechnology). Quantified protein $(20 \mu \mathrm{g})$ was separated by $10 \%$ sodium dodecyl sulfate-polyacrylamide gel electrophoresis (SDS-PAGE). The proteins were blotted onto a polyvinylidene difluoride membrane, and the membranes were soaked in $5 \%$ milk/PBS 
Table I. The sequences of the primers for RT-qPCR.

\begin{tabular}{lcc}
\hline Gene name & Forward (from 5' to 3') & Reverse (from 5' to 3') \\
\hline MMP2 & CAAGTTCCCCGGCGATGTC & CTGGACAGCCAGACACTAAAG \\
MMP9 & TTCTGGTCAAGGTCACCTGTC & CTCGCGGCAAGTCTTCAGAG
\end{tabular}

RT-qPCR, reverse transcription-quantitative polymerase chain reaction; MMP, matrix metalloproteinase.

with Tween-20 (PBST) for $1 \mathrm{~h}$. Subsequently, the membranes were incubated with primary antibodies, including p53 (dilution 1:800; cat. no. 2524), p27 (dilution 1:800; cat. no. 2552), apoptosis regulator $\mathrm{Bcl}-2$ (Bcl-2; dilution 1:800; cat. no. 4223), apoptosis regulator Bax (Bax; dilution 1:800; cat. no. 2772), caspase-3 (dilution 1:800; cat. no. 9662; all from Cell Signaling Technology, Inc., Danvers, MA, USA), p21 (dilution 1:600; cat. no. ab109520), cyclin D1 (dilution 1:500; cat. no. ab134175), matrix metalloproteinase (MMP)2 (dilution 1:1,000; cat.no.ab37150),MMP9 (dilution 1:200; cat.no.ab38898),PI3K (dilution 1:1,000; cat. no. ab191606), p-PI3K (dilution 1:500; cat. no. ab182651), AKT (dilution 1:500; cat. no. ab8805), p-AKT (dilution 1:2,000; cat. no. ab81283) and $\beta$-actin (dilution 1:2,000; cat. no. ab8226; all from Abcam, Cambridge, $\mathrm{UK})$ at $4^{\circ} \mathrm{C}$ overnight. Following washing with PBST three times, the membranes were incubated with HRP-conjugated goat anti-rabbit antibody (dilution 1:3,000; cat. no. ab6721) and goat anti-mouse antibody (dilution 1:3,000; cat. no. ab6789; both from Abcam) for $1 \mathrm{~h}$ at room temperature. Finally, the protein bands were visualized via enhanced chemiluminescence (Beyotime Institute of Biotechnology) and quantified by Image-Pro Plus 6.0 software (Media Cybernetics).

Reverse transcription-quantitative polymerase chain reaction $(R T-q P C R)$. The collected cells or tumor tissue were lysed in TRIzol ${ }^{\circledR}$ reagent (Thermo Fisher Scientific, Inc.) and the total RNA was extracted. cDNA was acquired by RT using a commercial kit (Thermo Fisher Scientific, Inc.). qPCR was performed to detect mRNA expression levels, including MMP2 and MMP9 using a SYBR-Green Kit (Thermo Fisher Scientific, Inc.). Thermocycling conditions for RT-qPCR were as follows: i) $50^{\circ} \mathrm{C}$ for $2 \mathrm{~min}, 1$ cycle; ii) $95^{\circ} \mathrm{C}$ for $10 \mathrm{~min}$, 1 cycle; iii) $95^{\circ} \mathrm{C}$ for $15 \mathrm{sec}, 60^{\circ} \mathrm{C}$ for $30 \mathrm{sec}$ and $72^{\circ} \mathrm{C}$ for $30 \mathrm{sec}, 40$ cycles. The comparative $\Delta \Delta \mathrm{Cq}$ method was used to quantify relative gene expression as previously descripted (18). The sequences of the primers are listed in Table I.

Immunohistochemistry. The tumor tissues were embedded in paraffin and cut into $5 \mu \mathrm{m}$ sections. Following dewaxing and rehydration, the slides were placed in boiling PBS for $10 \mathrm{~min}$ for antigen retrieval, followed by incubation of the sections with $0.3 \%$ hydrogen peroxide for $30 \mathrm{~min}$ at room temperature to block endogenous peroxidase activity. The sections were blocked by $0.5 \%$ BSA/PBST containing $10 \%$ goat serum for $60 \mathrm{~min}$ at room temperature. The slides were incubated with primary antibodies, including MMP2 (dilution 1:200; cat. no. ab37150), MMP9 (dilution 1:200; cat. no. ab38898), proliferating cell nuclear antigen (PCNA; dilution 1:400; cat. no. ab29; all from Abcam) and caspase-3 (dilution 1:200; cat. no. 9662; Cell Signaling Technology, Inc.) at $4^{\circ} \mathrm{C}$ overnight. Following three washes with PBS, the sections were incubated with HRP-conjugated goat anti-rabbit antibody (dilution 1:600; cat. no. ab6721) and goat anti-mouse antibody (dilution 1:600; cat. no. ab6789; both from Abcam) for $1 \mathrm{~h}$ at room temperature. The color was developed using the 3,5-diaminobenzidine (DAB; Vector Laboratories, Inc., Burlingame, CA, USA) substrate, followed by counterstaining with hematoxylin. The images were captured by a light microscope (Nikon).

ATP assay. The ATP content was determined in cell extracts using a luminescent ATP detection kit (ATPlite Luminescence Assay System; PerkinElmer Life Sciences, Waltham, MA, USA). The experiment was performed according to the manufacturer's protocol. The luminescence intensity was detected using a microplate reader.

Statistical analysis. Data are expressed as the means \pm standard deviation. For the comparison of 2 groups, a Student's t-test was used. When comparing $\geq 3$ groups, one-way analysis of variance (ANOVA) was used, if the ANOVA was significant, post hoc testing of differences between groups was performed using Fisher's least significant difference test. $\mathrm{P}<0.05$ was considered to indicate a statistically significant difference. The analysis was performed using SPSS 18.0 software (SPSS, Inc., Chicago, IL, USA).

\section{Results}

Effect of ISL on Saos-2 cell viability. To investigate whether ISL was able to specifically inhibit the cell viability of osteosarcoma cell line Saos-2, Saos-2 and MC3T3-E1 (mouse embryonic osteoblastic cells) cells were treated with different doses of ISL for $48 \mathrm{~h}$. The results demonstrated that when treated with $30 \mu \mathrm{M}$ ISL, Saos- 2 cell viability began to decrease significantly, while MC3T3-E1 cells remained unaltered (Fig. 1A). Subsequently, $30 \mu \mathrm{M}$ ISL was selected to treat Saos-2 cells across a number of time-points. The growth of ISL-treated Saos-2 cells was significantly inhibited at the indicated time-points compared with the control group, and the optimum inhibitory effect of ISL on Saos-2 cells was observed at $48 \mathrm{~h}$ (Fig. 1B).

Effect of ISL on Saos-2 cell proliferation. To investigate whether the decrease in Saos-2 cell viability induced by ISL was associated with cell cycle arrest, the cell cycle distribution was analyzed. The results demonstrated that ISL was able to 

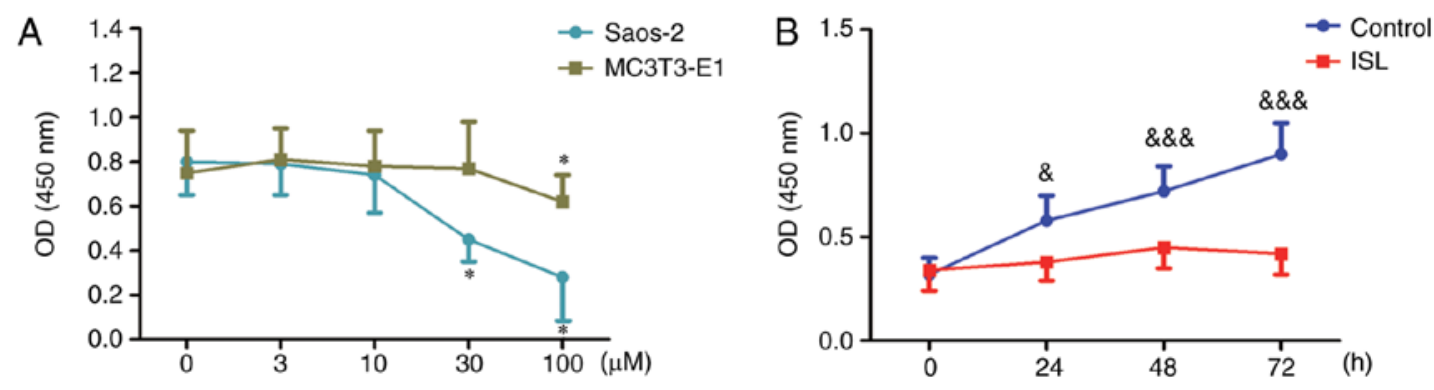

Figure 1. Effect of ISL on Saos-2 cell viability. (A) Cultured Saos-2 and MC3T3-E1 cells were incubated with various doses of ISL for $48 \mathrm{~h}$, and cell viability was determined by CCK-8 assay. (B) Cultured Saos-2 cells were incubated with or without $30 \mu \mathrm{M}$ ISL for the indicated time-points. Cell viability was determined by CCK-8 assay. ( ${ }^{*}<0.05$ vs. $0 \mu \mathrm{M},{ }^{\&} \mathrm{P}<0.05$, \& \&\& $\mathrm{P}<0.001$ vs. the control group; $\mathrm{n}=5$. ISL, isoliquiritigenin; CCK-8, Cell Counting Kit-8.
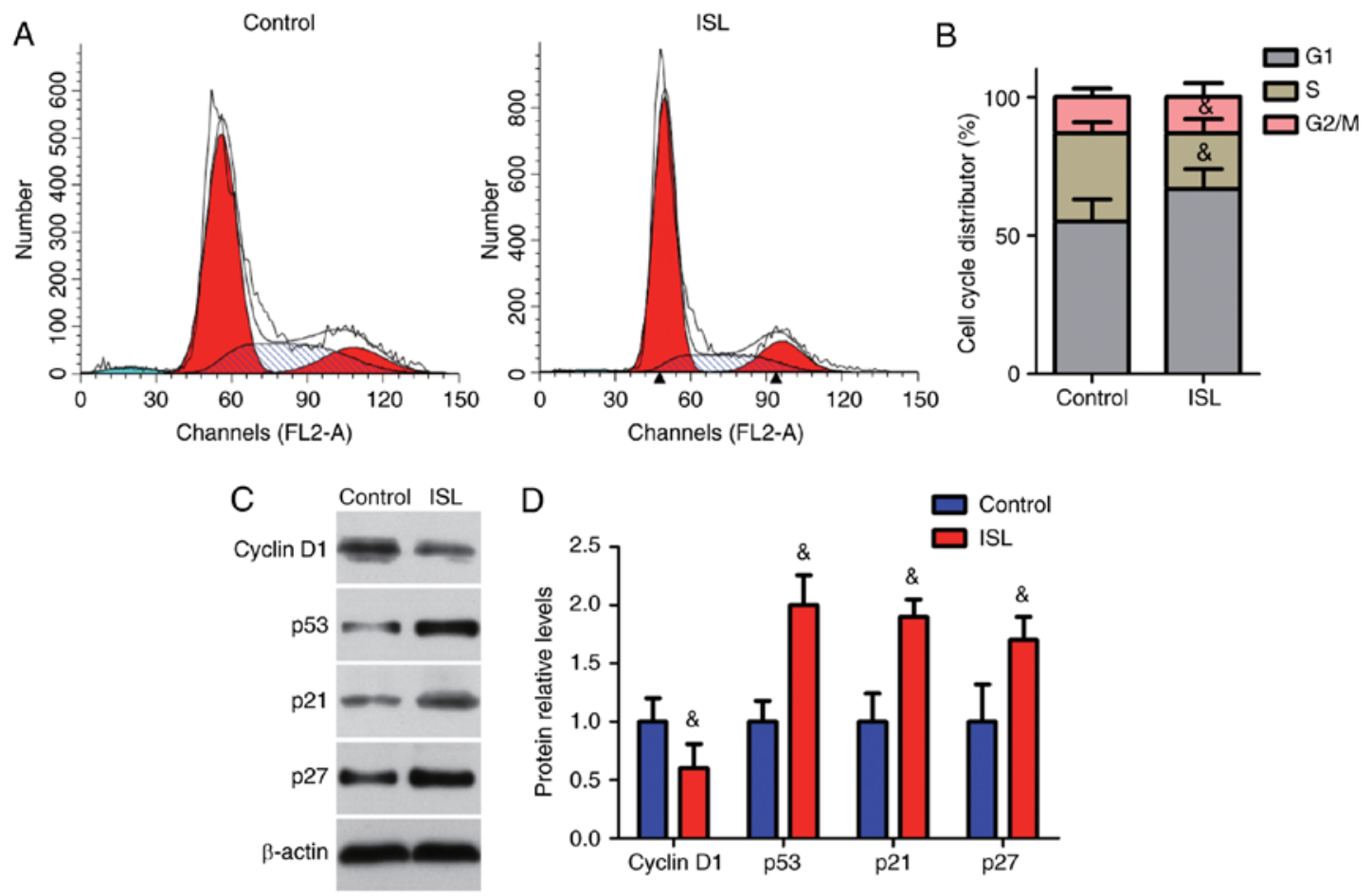

Figure 2. Effect of ISL on Saos-2 cell proliferation. Cultured Saos-2 cells were incubated with or without $30 \mu \mathrm{M}$ ISL for $48 \mathrm{~h}$. (A) Cell cycle analysis. (B) Quantification of cell cycle distribution. (C) Western blot analysis of the expression of cyclin D1, p53, p21 and p27. $\beta$-actin was used as a loading control.

(D) Quantification of the protein expression levels of cyclin $\mathrm{D} 1, \mathrm{p} 53, \mathrm{p} 21$ and $\mathrm{p} 27 .{ }^{\circledR} \mathrm{P}<0.05$ vs. the control group; $\mathrm{n}=5$. ISL, isoliquiritigenin.

significantly increase the number of cells in the G1 phase and decrease the number of cells in the S phase (Fig. 2A and B). Western blot analysis revealed that the expression of cyclin D1 was significantly downregulated, while p53, p21 and p27 were significantly upregulated in ISL-treated Saos-2 cells, compared with the control group (Fig. 2C and D).

Effect of ISL on Saos-2 cell apoptosis. To investigate whether ISL was able to induce Saos-2 cell apoptosis, Annexin V and PI staining was performed. The results demonstrated that the apoptosis rate in ISL-treated cells was significantly higher compared with that in the control cells (Fig. 3A and B). Further analysis revealed that ISL was able to significantly downregulate the expression of anti-apoptosis protein $\mathrm{Bcl}-2$ and upregulate the expression of the pro-apoptotic proteins Bax and caspase-3 (Fig. 3C and D). In addition, ATP production was significantly inhibited by ISL (Fig. 3E).
Effect of ISL on Saos-2 cell migration. To investigate whether ISL was able to inhibit Saos-2 cell migration, scratch test and Transwell assays were performed. To avoid the effects of cell viability of having an effect on the migration ability of the cells, an ISL dose that has no effect on cell viability is necessary and required for the cell migration assay. Since cell viability was significantly reduced when Saos-2 cells were treated with ISL at a dose of $30 \mu \mathrm{M}, 10 \mu \mathrm{M}$ ISL was used to treat cells. The results demonstrated that the cell migration distance was shorter, while the number of migratory cells was decreased in the ISL group compared with the control group (Fig. 4A-D). Furthermore, the protein and mRNA expression levels of MMP2 and MMP9 in Saos-2 cells were downregulated by treatment with ISL (Fig. 4E-G).

Effect of ISL on the PI3K/AKT pathway in Saos-2 cells. To investigate whether ISL affected the expression of 

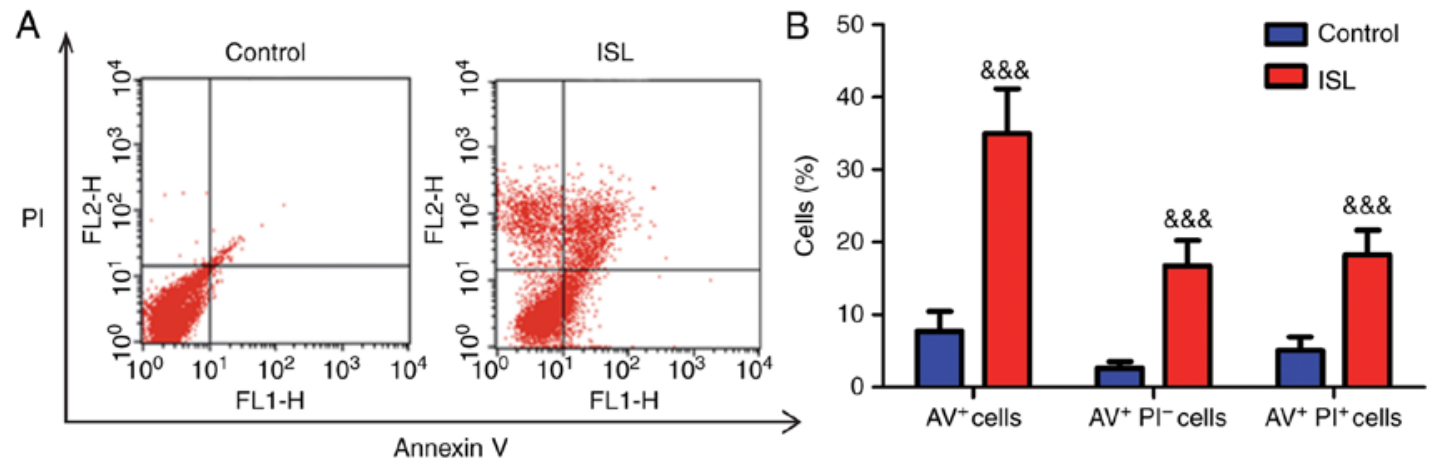

C
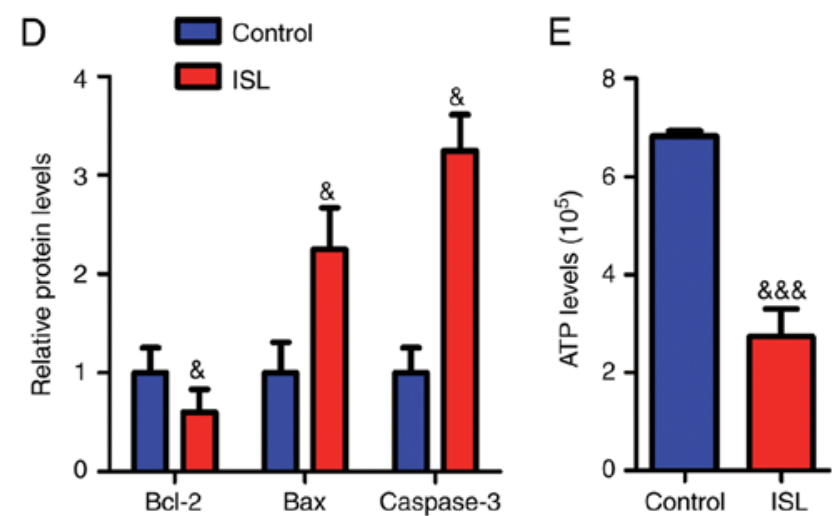

Figure 3. Effect of ISL on Saos-2 cell apoptosis. Cultured Saos-2 cells were incubated with or without $30 \mu \mathrm{M}$ ISL for $48 \mathrm{~h}$. (A) Cell apoptosis was determined by staining with Annexin V and PI. (B) Quantification of cell apoptosis. (C) Western blot analysis of the expression of Bcl-2, Bax and caspase-3. $\beta$-actin was used as a loading control. (D) Quantification of the protein expression levels of Bcl-2, Bax and caspase-3. (E) Intracellular ATP production assay. ${ }^{\&} \mathrm{P}<0.05$, \&\&\& $\mathrm{P}<0.001$ vs. the control group; $\mathrm{n}=5$. ISL, isoliquiritigenin; PI, propidium iodide; Bcl-2, apoptosis regulator Bcl-2; Bax, apoptosis regulator Bax.
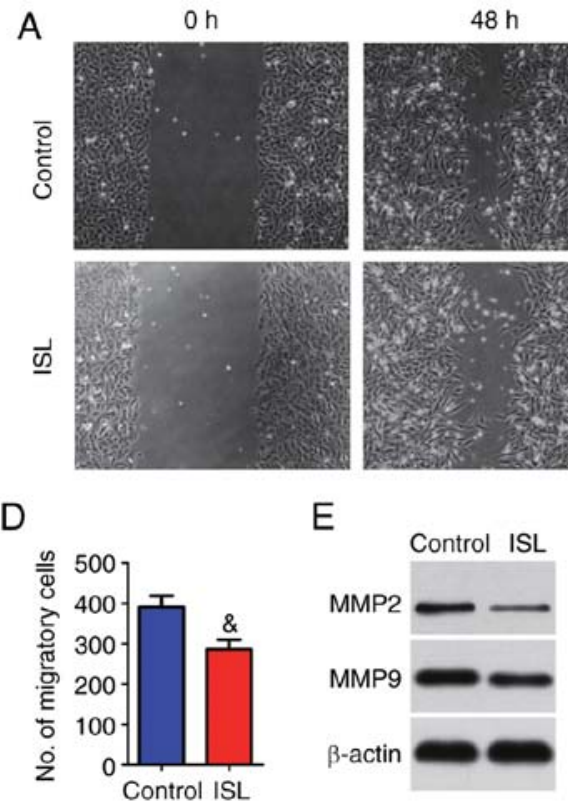
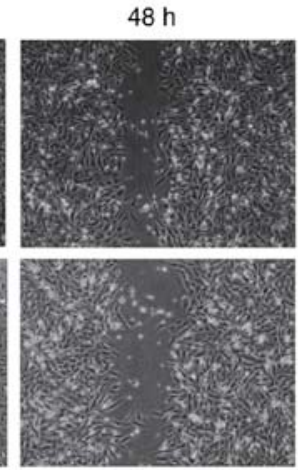

B

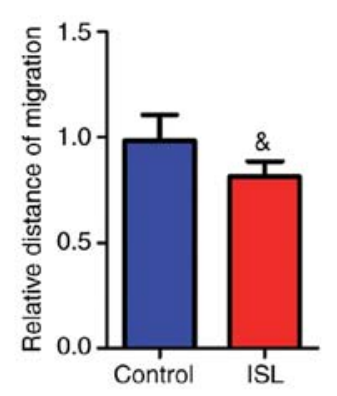

E

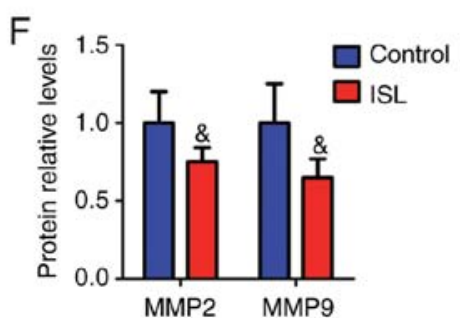

C

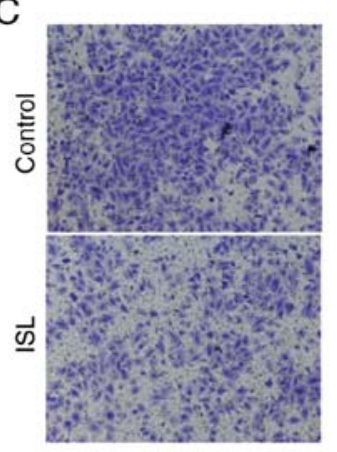

G

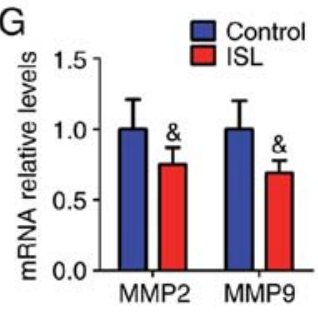

Figure 4. Effect of ISL on Saos-2 cell migration. Cultured Saos-2 cells were incubated with or without $10 \mu \mathrm{M}$ ISL for $48 \mathrm{~h}$. (A) Representative images of the scratch test at 0 and $48 \mathrm{~h}$. Magnification, x100. (B) Quantification of the cell migration distance. (C) Representative images of migratory cells stained with crystal violet. Magnification, $x 100$. (D) The number of migratory cells. (E) Western blot analysis of the expression of MMP2 and MMP9. $\beta$-actin was used as a loading control. (F) Quantification of the protein expression levels of MMP2 and MMP9. (G) Reverse transcription-quantitative polymerase chain reaction analysis of the mRNA expression levels of MMP2 and MMP9. \& $\mathrm{P}<0.05$ vs. the control group; $n=5$. ISL, isoliquiritigenin; MMP, matrix metalloproteinase.

molecules in the PI3K/AKT pathway, the expression of associated proteins was assessed. The results demonstrated that although the protein expression levels of total PI3K and
AKT were not affected by treatment with ISL, the expression levels of p-PI3K and p-AKT were significantly downregulated (Fig. 5A and B). 

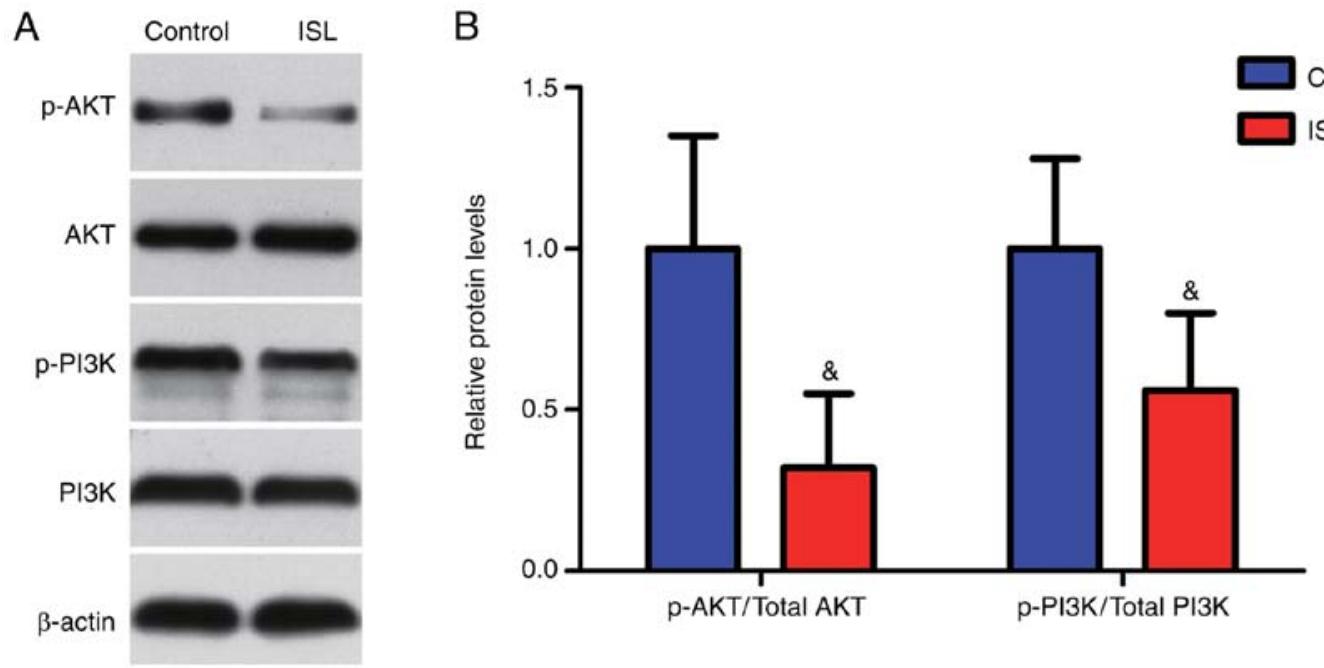

Figure 5. Effect of ISL on the PI3K/AKT pathway in Saos-2 cells. Cultured Saos-2 cells were incubated with or without $30 \mu \mathrm{M}$ ISL for $48 \mathrm{~h}$. (A) Western blot analysis of the expression of AKT, p-AKT, PI3K and p-PI3K. $\beta$-actin was used as a loading control. (B) Quantification of the protein expression levels of AKT, p-AKT and PI3K, p-PI3K. ${ }^{\circledR} \mathrm{P}<0.05$ vs. the control group, n=5. ISL, isoliquiritigenin; PI3K, phosphatidylinositol 3-kinase; AKT, RAC- $\alpha$ serine/threonine-protein kinase.
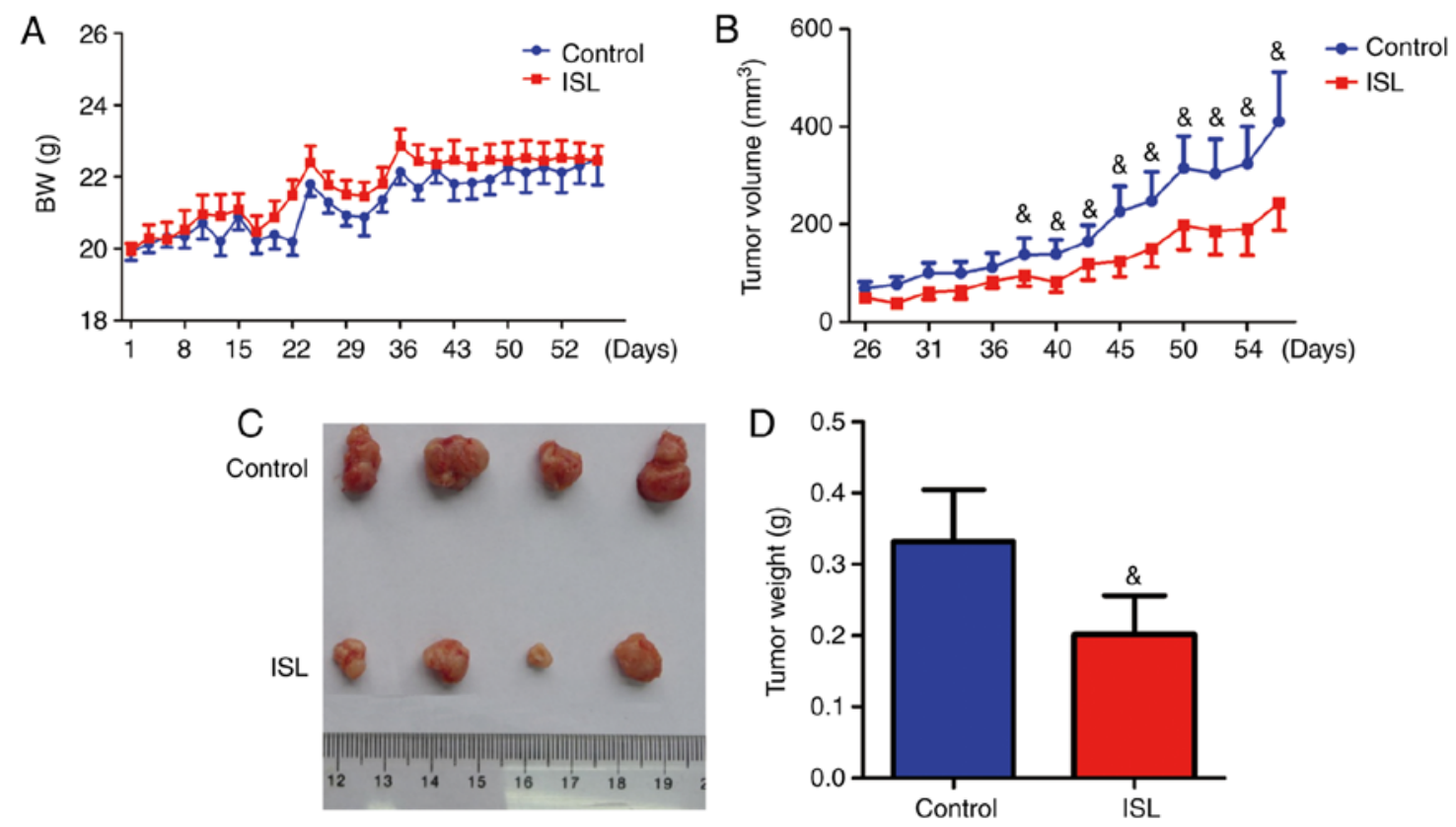

Figure 6. Effect of ISL on the growth of xenograft tumors. The mice were treated with corn oil or ISL ( $50 \mathrm{mg} / \mathrm{kg} / \mathrm{day})$. (A) The body weight of ISL-treated or corn oil-treated mice. (B) Tumor volume. (C) Tumor size. (D) Tumor weight. ${ }^{\circledR} \mathrm{P}<0.05$ vs. the control group; $\mathrm{n}=5$. ISL, isoliquiritigenin.

Effect of ISL on the growth of xenograft tumors. To investigate whether ISL was able to suppress the growth of xenograft tumors, Saos-2 cells were implanted via subcutaneous injection into the flank of ISL-treated or control mice. Body weight and tumor volume were monitored. The results demonstrated that treatment with ISL had no influence on body weight (Fig. 6A). However, the ISL-treated tumor size began to significantly decrease at day 40 post-injection, compared with vehicle-treated tumors (Fig. 6B). Following sacrifice, the tumors were removed, and the tumor size and weight were compared between vehicle-treated and ISL-treated mice. The tumor size and tumor weight were decreased in ISL-treated mice compared with control mice (Fig. 6C and D).
Effect of ISL on cell proliferation, apoptosis and migration in the xenograft tumor model. To investigate the effect of ISL on cell proliferation, cell migration and cell apoptosis in the xenograft tumor model, PCNA, caspase-3, MMP2 and MMP9 immunohistochemistry was performed on the tumor sections. The results demonstrated that PCNA, MMP2 and MMP9 positive cells were significantly reduced and caspase- 3 positive cells were significantly increased in the ISL-treated tumors compared with the control tumors (Fig. 7A-H). Western blot analysis revealed that the expression of total PI3K and AKT in ISL-treated and control tumors was similar, although the levels of p-PI3K and p-AKT were significantly decreased in ISL-treated tumors (Fig. 7I and J). 
A

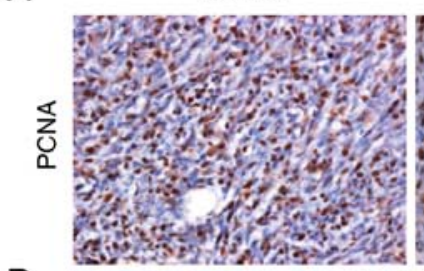

B

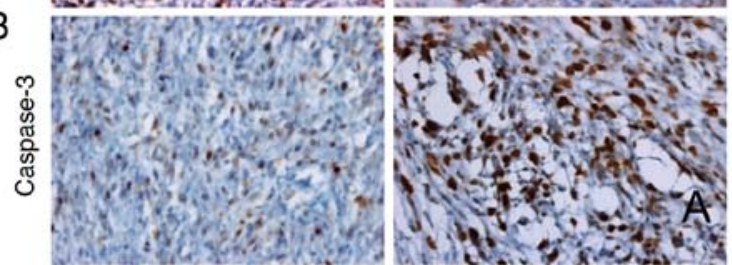

ISL

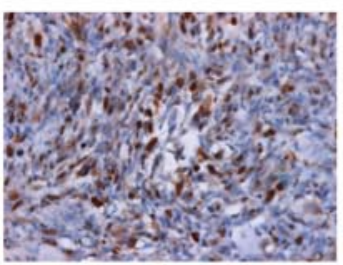

C

\section{$\sum_{\Sigma}^{\Sigma}$}

D
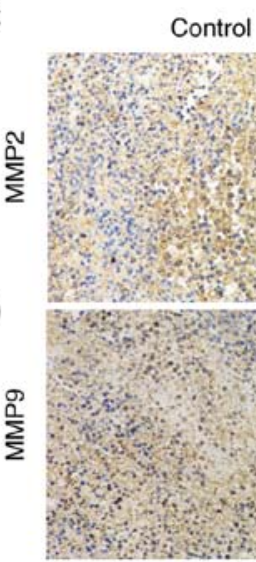

G

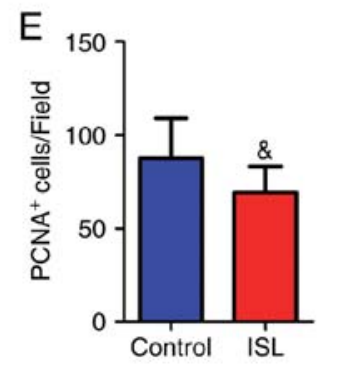

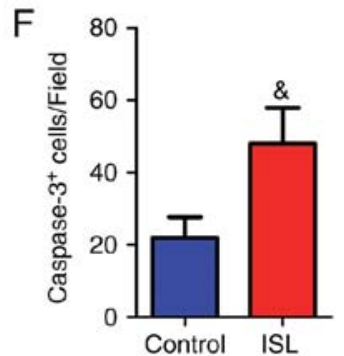

Control
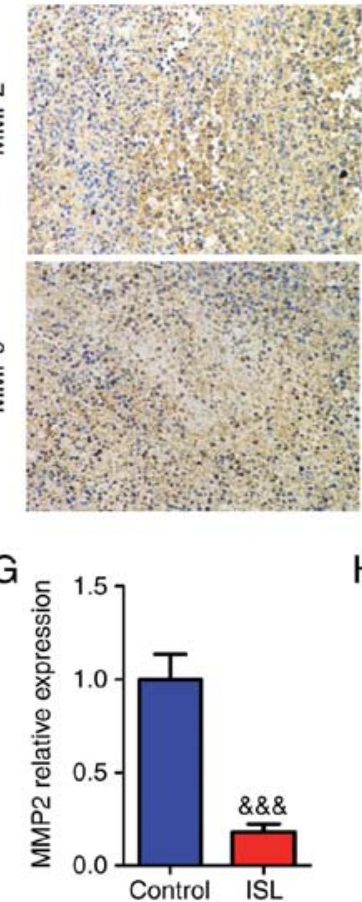

ISL

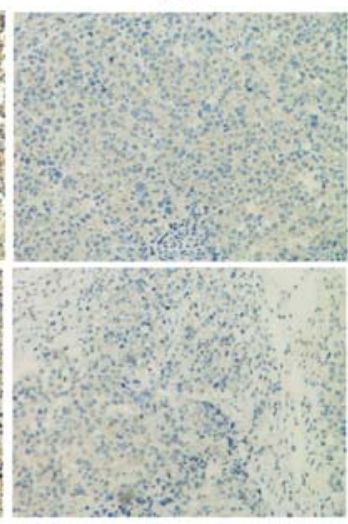

$\mathrm{H}$

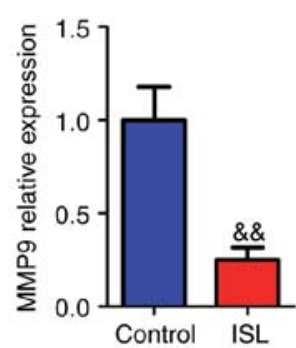

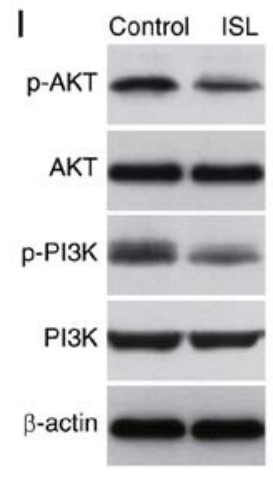

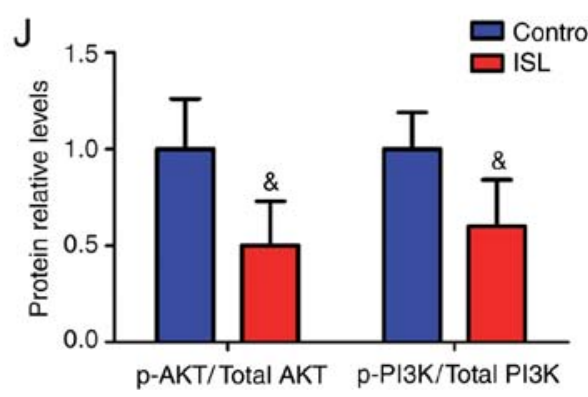

Figure 7. Effect of ISL on cell proliferation, apoptosis and migration in xenograft tumors of Saos-2 cells. Immunohistochemistry on sections of tumors with antibodies against (A) PCNA, (B) caspase-3, (C) MMP2 and (D) MMP9. Magnification is x400 in A and B, and x200 in C and D. Quantification of (E) PCNA and (F) caspase-3-positive cells. Relative expression of (G) MMP2 and (H) MMP9. (I) Western blot analysis of the expression of AKT, p-AKT, PI3K and p-PI3K. $\beta$-actin was used as a loading control. (J) Quantification of the protein expression levels of AKT, p-AKT, PI3K and p-PI3K. ${ }^{\text {\& }} \mathrm{P}<0.05$, \&\&\& $<0.01$,

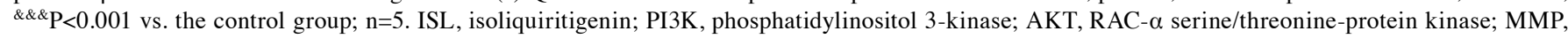
matrix metalloproteinase; PCNA, proliferating cell nuclear antigen.

\section{Discussion}

In the present study, the effect of ISL on the growth of osteosarcoma was investigated. Since, compared with other osteosarcoma cell lines including MG-63 or U2OS, Saos-2 cells exhibit better tumorigenesis potential in vivo, which benefits the in vivo study, Saos-2 cells were used in the present study. It was demonstrated that ISL was able to suppress the growth of the osteosarcoma cell line Saos-2 cells in vitro and in vivo. This inhibitory effect of ISL was associated with decreasing cell proliferation and increasing cell apoptosis. Furthermore, the in vitro experiments demonstrated that ISL was able to inhibit the migratory potential of Saos-2 cells. The underlying mechanisms of action of ISL in osteosarcoma may depend on the inhibitory effect of ISL on the PI3K/AKT signaling pathway.
Rapid cell division is one of the most important hallmarks of tumors. In cells, cell division is promoted by cyclin-dependent kinases (CDKs) interacting with cyclins (19). This interaction may be blocked by cell cyclin-dependent kinase inhibitors (CDKIs) (20). In the present study, it was revealed that ISL was able to significantly upregulate the expression of p53, p21 and p27 and downregulated cyclin D1 in cultured Saos-2 cells. Furthermore, ISL inhibited the proliferation of Saos-2 cells in vivo. These results are consistent with previous reports investigating the antiproliferative effect of ISL in other types of cancer (7,21-23). Therefore, the suppressive effect of ISL on osteosarcoma is partly mediated by inhibition of cell division.

Increasing evidence suggests that ISL is a potent apoptosis inducer in tumor cells (24-27). The present study demonstrated that ISL was able to induce the apoptosis of Saos-2 
cells in vitro and in xenograft tumors. Previous studies have demonstrated that ISL-triggered cell apoptosis is associated with disruption of mitochondrial function (28-30). It is generally acknowledged that mitochondria serve a critical role in mediating cell apoptosis. A cluster of proteins belonging to the Bcl-2 family localize to the outer membrane of mitochondria to regulate cell apoptosis (31). The Bcl-2 family proteins may be classified into two groups: One is the anti-apoptotic proteins, including $\mathrm{Bcl}-2$, and the other is the pro-apoptotic proteins including Bax. The balance of these two proteins determines whether a cell undergoes apoptosis $(32,33)$. In the present study, it was observed that ISL downregulated the expression of the anti-apoptotic protein Bcl-2 and upregulated the expression of the pro-apoptotic protein Bax in cultured Saos-2 cells. Consistent with this, ATP synthesis which occurs primarily in mitochondria was significantly inhibited by ISL. Therefore, ISL induced osteosarcoma apoptosis via mitochondrial signaling. A previous study demonstrated that ISL induced the apoptosis of Hep-G2 cells in a p53-dependent manner (34). In the present study, the upregulation of p53 may have been involved in promoting osteosarcoma apoptosis. In conclusion, ISL-induced cell apoptosis partly accounted for the osteosarcoma growth-inhibitory effect of ISL.

It has been noted that ISL may inhibit the metastasis of various tumors $(13,35)$. In the present study, it was demonstrated that ISL inhibited the migratory potential of cultured Saos-2 cells. Breakdown of the extracellular matrix (ECM) is a crucial event in tumor cell invasion. This process is regulated by a number of molecules, particularly MMP2, MMP9 and tissue inhibitors of metalloproteinases (TIMPs) (36-38). It has been demonstrated that MMP2 and MMP9 are required for osteosarcoma metastasis $(39,40)$. A previous study demonstrated that ISL inhibits the metastasis of breast cancer by downregulating the expression of MMP2 and MMP9 (13). Consistent with this finding, it was observed that ISL downregulated the expression of MMP2 and MMP9 in cultured Saos- 2 cells and in xenograft tumors.

One critical underlying mechanism of the biological function of ISL is its ability to modify the intracellular PI3K/AKT pathway. It has been demonstrated that ISL induces melanin degradation in human epidermal keratinocytes and inhibits the proliferation of human arterial smooth muscle cells by suppressing PI3K/AKT signaling $(41,42)$. In addition, numerous studies have demonstrated that ISL may inhibit the phosphorylation of PI3K and AKT in tumor cells, thereby suppressing the growth of the tumor (11-13). In the present study, it was demonstrated that ISL was able to significantly reduce the levels of $\mathrm{p}$-AKT and $\mathrm{p}-\mathrm{PI} 3 \mathrm{~K}$ in Saos- 2 cells in vitro and in xenograft tumors. It has previously been demonstrated that the PI3K/AKT pathway is vital for the initiation and progression of osteosarcoma (16). A broad spectrum of molecules involved in cell proliferation, survival and invasion are directly or indirectly regulated by PI3K/AKT signaling (16). Drugs targeting PI3K/AKT signaling have been developed against osteosarcoma, including PI3K pan inhibitors. However, the safety of these drugs is undergoing evaluation and remains controversial. In the present study, it was revealed that treatment with ISL was associated with low cytotoxicity in MC3T3-E1 cells and had no influence on the body weight of mice, indicating that ISL may be a safe drug for osteosarcoma treatment. This result is consistent with a previous study reporting that ISL may significantly decrease the tumor size of mice without evident weight loss (17).

In conclusion, it was demonstrated that ISL was able to inhibit cell proliferation and induce the cell apoptosis of osteosarcoma in vitro and in vivo, possibly by deactivating the PI3K/AKT signaling pathway. In addition, it was also demonstrated that ISL attenuated the migration of cultured Saos- 2 cells. The present study indicated that ISL may serve as a potential drug for osteosarcoma treatment.

\section{Acknowledgements}

Not applicable.

\section{Funding}

This study was supported by the China Postdoctoral Science Foundation Funded Project (grant no. 2016M593039).

\section{Availability of data and materials}

All data generated or analyzed during the present study are included in this published article.

\section{Authors' contributions}

SW and XS conceived and designed the study. CL, XZ, CS and XL performed the experiments and analyzed the data. CL and XZ wrote the paper. SW and XS reviewed and edited the manuscript. CL, CS and XL performed the experiments required for revision. All authors read and approved the manuscript and agree to be accountable for all aspects of the research in ensuring that the accuracy or integrity of any part of the work are appropriately investigated and resolved.

\section{Ethics approval and consent to participate}

All animal experiments were approved by the Committee on the Ethics of Animal Experiments of Nanjing University and were performed strictly in accordance with the recommendations in the Guide for the Care and Use of Laboratory Animals of the National Institutes of Health (Bethesda, MD, USA).

\section{Patient consent for publication}

Not applicable.

\section{Competing interests}

The authors declare that they have no competing interests.

\section{References}

1. Zambo I and Vesely K: WHO classification of tumours of soft tissue and bone 2013: The main changes compared to the 3rd edition. Cesk Patol 50: 64-70, 2014 (In Czech).

2. Bernthal NM, Federman N, Eilber FR, Nelson SD, Eckardt JJ, Eilber FC and Tap WD: Long-term results ( $>25$ years) of a randomized, prospective clinical trial evaluating chemotherapy in patients with high-grade, operable osteosarcoma. Cancer 118: 5888-5893, 2012 . 
3. Eilber FR and Rosen G: Adjuvant chemotherapy for osteosarcoma. Semin Oncol 16: 312-322, 1989.

4. Chin YW, Jung HA, Liu Y, Su BN, Castoro JA, Keller WJ, Pereira MA and Kinghorn AD: Anti-oxidant constituents of the roots and stolons of licorice (Glycyrrhiza glabra). J Agric Food Chem 55: 4691-4697, 2007.

5. Peng F, Du Q, Peng C, Wang N, Tang H, Xie X, Shen J and Chen J: A review: The pharmacology of isoliquiritigenin. Phytother Res 29: 969-977, 2015.

6. Asl MN and Hosseinzadeh $\mathrm{H}$ : Review of pharmacological effects of Glycyrrhiza sp. and its bioactive compounds. Phytother Res 22: 709-724, 2008.

7. Lin Y, Sun H, Dang Y and Li Z: Isoliquiritigenin inhibits the proliferation and induces the differentiation of human glioma stem cells. Oncol Rep 39: 687-694, 2018.

8. Chen HY, Huang TC, Shieh TM, Wu CH, Lin LC and Hsia SM: Isoliquiritigenin induces autophagy and inhibits ovarian cancer cell growth. Int J Mol Sci 18: pii: E2025, 2017.

9. Hsu YL, Kuo PL, Lin LT and Lin CC: Isoliquiritigenin inhibits cell proliferation and induces apoptosis in human hepatoma cells. Planta Med 71: 130-134, 2005.

10. Wang Z, Wang N, Han S, Wang D, Mo S, Yu L, Huang H, Tsui K, Shen $\mathbf{J}$ and Chen J: Dietary compound isoliquiritigenin inhibits breast cancer neoangiogenesis via VEGF/VEGFR-2 signaling pathway. PLoS One 8: e68566, 2013.

11. Jung JI, Chung E, Seon MR, Shin HK, Kim EJ, Lim SS, Chung WY, Park KK and Park JH: Isoliquiritigenin (ISL) inhibits ErbB3 signaling in prostate cancer cells. Biofactors 28: 159-168, 2006.

12. Li Y, Zhao H, Wang Y, Zheng H, Yu W, Chai H, Zhang J, Falck JR, Guo AM, Yue J, et al: Isoliquiritigenin induces growth inhibition and apoptosis through downregulating arachidonic acid metabolic network and the deactivation of PI3K/Akt in human breast cancer. Toxicol Appl Pharmacol 272: 37-48, 2013.

13. Wang KL, Hsia SM, Chan CJ, Chang FY, Huang CY, Bau DT and Wang PS: Inhibitory effects of isoliquiritigenin on the migration and invasion of human breast cancer cells. Expert Opin Ther Targets 17: 337-349, 2013.

14. Zhu J, Sun Y, Lu Y, Jiang X, Ma B, Yu L, Zhang J, Dong X and Zhang Q: Glaucocalyxin A exerts anticancer effect on osteosarcoma by inhibiting GLI1 nuclear translocation via regulating PI3K/Akt pathway. Cell Death Dis 9: 708, 2018.

15. Angulo P, Kaushik G, Subramaniam D, Dandawate P, Neville K, Chastain K and Anant S: Natural compounds targeting major cel signaling pathways: A novel paradigm for osteosarcoma therapy. J Hematol Oncol 10: 10, 2017.

16. Zhang J, Yu XH, Yan YG, Wang $\mathrm{C}$ and Wang WJ: PI3K/Akt signaling in osteosarcoma. Clin Chim Acta 444: 182-192, 2015.

17. Wu CH, Chen HY, Wang CW, Shieh TM, Huang TC, Lin LC, Wang KL and Hsia SM: Isoliquiritigenin induces apoptosis and autophagy and inhibits endometrial cancer growth in mice. Oncotarget 7: 73432-73447, 2016.

18. Livak KJ and Schmittgen TD: Analysis of relative gene expression data using real-time quantitative PCR and the $2^{-\Delta \Delta C_{\mathrm{T}}}$ method. Methods 25: 402-408, 2001.

19. Ingham M and Schwartz GK: Cell-cycle therapeutics come of age. J Clin Oncol 35: 2949-2959, 2017.

20. Akin S, Babacan T, Sarici F and Altundag K: A novel targeted therapy in breast cancer: Cyclin dependent kinase inhibitors. J Buon 19: 42-46, 2014.

21. Wang Y, Ma J, Yan X, Chen X, Si L, Liu Y, Han J, Hao W and Zheng Q: Isoliquiritigenin inhibits proliferation and induces apoptosis via alleviating hypoxia and reducing glycolysis in mouse melanoma B16F10 cells. Recent Pat Anticancer Drug Discov 11: 215-227, 2016.

22. Zhang X, Yeung ED, Wang J, Panzhinskiy EE, Tong C, Li W and $\mathrm{Li} \mathrm{J}$ : Isoliquiritigenin, a natural anti-oxidant, selectively inhibits the proliferation of prostate cancer cells. Clin Exp Pharmacol Physiol 37: 841-847, 2010.
23. Maggiolini M, Statti G, Vivacqua A, Gabriele S, Rago V, Loizzo M, Menichini F and Amdò S: Estrogenic and antiproliferative activities of isoliquiritigenin in MCF7 breast cancer cells. J Steroid Biochem Mol Biol 82: 315-322, 2002.

24. Li ZX, Li J, Li Y, You K, Xu H and Wang J: Novel insights into the apoptosis mechanism of DNA topoisomerase I inhibitor isoliquiritigenin on HCC tumor cell. Biochem Biophys Res Commun 464: 548-553, 2015.

25. Jung SK, Lee MH, Lim DY, Kim JE, Singh P, Lee SY, Jeong CH, Lim TG, Chen H, Chi YI, et al: Isoliquiritigenin induces apoptosis and inhibits xenograft tumor growth of human lung cancer cells by targeting both wild type and L858R/T790M mutant EGFR. J Biol Chem 289: 35839-35848, 2014.

26. Hirchaud F, Hermetet F, Ablise M, Fauconnet S, Vuitton DA, Prétet JL and Mougin C: Isoliquiritigenin induces caspase-dependent apoptosis via downregulation of HPV16 E6 expression in cervical cancer Ca Ski cells. Planta Med 79: 1628-1635, 2013.

27. Chen G, Hu X, Zhang W, Xu N, Wang FQ, Jia J, Zhang WF, Sun ZJ and Zhao YF: Mammalian target of rapamycin regulates isoliquiritigenin-induced autophagic and apoptotic cell death in adenoid cystic carcinoma cells. Apoptosis 17: 90-101, 2012.

28. Yuan X, Zhang B, Gan L, Wang ZH, Yu BC, Liu LL, Zheng QS and Wang ZP: Involvement of the mitochondrion-dependent and the endoplasmic reticulum stress-signaling pathways in isoliquiritigenin-induced apoptosis of HeLa cell. Biomed Environ Sci 26: 268-276, 2013

29. Jung JI, Lim SS, Choi HJ, Cho HJ, Shin HK, Kim EJ, Chung WY, Park KK and Park JH: Isoliquiritigenin induces apoptosis by depolarizing mitochondrial membranes in prostate cancer cells. J Nutr Biochem 17: 689-696, 2006.

30. Yang HH, Zhang C, Lai SH, Zeng CC, Liu YJ and Wang XZ: Isoliquiritigenin induces cytotoxicity in PC-12 cells in vitro. Appl Biochem Biotechnol 183: 1173-1190, 2017.

31. Kale J, Osterlund EJ and Andrews DW: BCL-2 family proteins: Changing partners in the dance towards death. Cell Death Differ 25: 65-80, 2018.

32. Maes ME, Schlamp CL and Nickells RW: BAX to basics: How the $B C L 2$ gene family controls the death of retinal ganglion cells. Prog Retin Eye Res 57: 1-25, 2017.

33. Strasser A and Vaux DL: Viewing BCL2 and cell death control from an evolutionary perspective. Cell Death Differ 25: 13-20, 2018.

34. Hsu YL, Kuo PL and Lin CC: Isoliquiritigenin induces apoptosis and cell cycle arrest through p53-dependent pathway in Hep G2 cells. Life Sci 77: 279-292, 2005.

35. Kwon GT, Cho HJ, Chung WY, Park KK, Moon A and Park JH: Isoliquiritigenin inhibits migration and invasion of prostate cancer cells: Possible mediation by decreased JNK/AP-1 signaling. J Nutr Biochem 20: 663-676, 2009.

36. Chien MH, Lin CW, Cheng CW, Wen YC and Yang SF: Matrix metalloproteinase-2 as a target for head and neck cancer therapy. Expert Opin Ther Targets 17: 203-216, 2013.

37. Kessenbrock K, Plaks V and Werb Z: Matrix metalloproteinases: Regulators of the tumor microenvironment. Cell 141: 52-67,2010.

38. Yang JS, Lin CW, Su SC and Yang SF: Pharmacodynamic considerations in the use of matrix metalloproteinase inhibitors in cancer treatment. Expert Opin Drug Metab Toxicol 12: 191-200, 2016.

39. Lan H, Hong W, Fan P, Qian D, Zhu J and Bai B: Quercetin inhibits cell migration and invasion in human osteosarcoma cells. Cell Physiol Biochem 43: 553-567, 2017.

40. Kunz P, Sahr H, Lehner B, Fischer C, Seebach E and Fellenberg J: Elevated ratio of MMP2/MMP9 activity is associated with poor response to chemotherapy in osteosarcoma. BMC Cancer 16: 223, 2016

41. Yang Z, Zeng B, Pan Y, Huang P and Wang C: Autophagy participates in isoliquiritigenin-induced melanin degradation in human epidermal keratinocytes through PI3K/AKT/mTOR signaling. Biomed Pharmacother 97: 248-254, 2018.

42. Chen T, Deng S and Lin R: The inhibitory effect of Isoliquiritigenin on the proliferation of human arterial smooth muscle cell. BMC Pharmacol Toxicol 18: 57, 2017. 